\title{
GLAD!
}

Revue sur le langage, le genre, les sexualités

11 | 2021

Archives, genre, sexualités, discours

\section{Archives de la diplomatie linguistique : explorer les rôles des femmes pour réécrire l'histoire?}

The Archives of Linguistic Diplomacy: Women out of the Past?

\section{Clémentine Rubio}

\section{(2) OpenEdition}

Journals

Édition électronique

URL : https://journals.openedition.org/glad/3170

DOI : $10.4000 /$ glad.3170

ISSN : 2551-0819

Éditeur

Association GSL

\section{Référence électronique}

Clémentine Rubio, «Archives de la diplomatie linguistique : explorer les rôles des femmes pour réécrire l'histoire? », GLAD! [En ligne], 11 | 2021, mis en ligne le 20 janvier 2021, consulté le 29 janvier 2022. URL : http://journals.openedition.org/glad/3170; DOI : https://doi.org/10.4000/glad.3170

Ce document a été généré automatiquement le 29 janvier 2022.

\section{(c) (†) $\ominus$}

La revue GLAD! est mise à disposition selon les termes de la Licence Creative Commons Attribution Pas d'Utilisation Commerciale - Pas de Modification 4.0 International. 


\section{Archives de la diplomatie linguistique : explorer les rôles des femmes pour réécrire l'histoire?}

The Archives of Linguistic Diplomacy: Women out of the Past?

\section{Clémentine Rubio}

1 Cet article, inscrit dans une recherche portant sur la diplomatie linguistique de la France, porte sur la place des femmes dans ce champ. Le thème de ce numéro, qui interroge les rapports des archives et du genre, constitue une occasion d'interroger la fabrique de l'histoire (de Certeau cité par Dosse 2006: 30). Le champ de la diplomatie linguistique française a majoritairement été étudié par le biais d'institutions ${ }^{1}$. Cet ancrage institutionnel tend à mettre en avant l'action d'hommes, en particulier lorsque l'on adopte une perspective historique. Les fonctions de diplomates, directeurs d'instituts, ministres sont en effet uniquement masculines jusqu'à une période récente. Les archives de la diplomatie linguistique donnent à voir cette réalité socioprofessionnelle. Pourtant, des traces de femmes existent bien dans ces archives. Cette étude, qui vise à interroger le rôle joué par les femmes dans la diplomatie linguistique, permet de souligner l'importance des archives dans la valorisation de ces rôles et dans l'écriture d'une histoire au masculin.

2 Il s'agira ainsi, à partir de quelques exemples issus de deux types de sources, d'interroger la manière dont les femmes apparaissent ou non, la manière dont leurs rôles sont ou ne sont pas décrits, transmis, valorisés, archivés; et ce que les archives font à ces rôles. 


\title{
S'adresser aux archives de la diplomatie linguistique par le prisme du genre
}

\author{
Diplomatie culturelle, diplomatie linguistique
}

3 Il convient de préciser ce qui sera entendu par «diplomatie linguistique » dans cet article. Anne Gazeau-Secret, dans son article Soft power, l'influence par la langue et la culture (2013) décrit la notion de diplomatie culturelle comme étant historiquement une spécificité française. La notion aurait été développée à la fin du XIX siècle, dans la même période que celle de francophonie et dans la période de création d'institutions ayant participé à la diffusion de la langue et de la culture françaises (on peut penser à l'Alliance israélite universelle, à l'Alliance française, à la Mission laïque française). Ce terme de «diplomatie culturelle» permet à la fois de souligner l'importance du domaine culturel mais aussi le poids de l'État dans ces stratégies. Pourquoi proposer, aux côtés des termes de "diplomatie culturelle ", ceux de "diplomatie linguistique " (Rubio 2018) ? Une des raisons tient au fait que le terme de "diplomatie culturelle » cache parfois l'accent mis sur la diffusion de la langue, et qui mérite un travail spécifique. On peut prendre pour exemple les centres culturels français et le poids qu'y pèsent les cours de français, en comparaison avec les autres activités (conférences, bibliothèques, manifestations culturelles). La diplomatie linguistique mérite une attention particulière $d u$ fait du produit singulier qu'elle diffuse et sur lequel elle repose. La langue est à la fois vecteur et objet d'enseignement et d'apprentissage, vecteur et objet de diffusion.

4 Cette recherche sur les femmes dans les archives de la diplomatie linguistique a été menée à partir de deux types d'archives : des documents d'archives diplomatiques, et les archives de l'EPPFE (École de préparation des professeurs de français à l'étranger). Celles-ci ne sont que rarement exploitées en didactique (à l'exception des travaux de la SIHFLES $^{2}$ et du projet CLIODIL $^{3}$ ) et en sociolinguistique ; elles présentent pourtant un intérêt important pour ces deux champs d'études sur le langage.

\section{Archives diplomatiques}

5 Ces archives proviennent des différents postes diplomatiques français à l'étranger (ambassades, consulats, vice-consulats) ainsi que des archives du ministère des Affaires étrangères rapatriées dans les centres d'archives de Nantes (pour les archives de postes diplomatiques) et de La Courneuve (pour les archives « centrales » du ministère).

On reviendra par la suite sur l'impact des politiques d'archivage sur la visibilité des femmes, il semble par conséquent important de préciser comment sont organisées ces archives et l'effet de cette organisation sur les projets de recherche. Au sein du Centre des archives diplomatiques de Nantes (CADN), les archives sont classées par postes (Jérusalem, Beyrouth...), puis par thématiques, et à l'intérieur des thématiques parfois par destinataire/expéditeur, et souvent par ordre chronologique. Les archives des postes consulaires retenues pour cet article émanent du poste du consulat général de France à Jérusalem. Elles avaient été sélectionnées et consultées pour une recherche doctorale pour laquelle avaient été ciblés les documents relevant des indexations " affaires religieuses ", « enseignement ", " affaires juives ", " relations à des consulats étrangers » à la recherche de ce que l'on disait de l'enseignement, de la langue, de la 
relation à l'Autre. Il semble important de préciser que le présent article ne s'appuie par conséquent pas sur des archives qui avaient initialement été explorées par le prisme du genre, mais par le prisme d'un questionnement sur les acteur.ices et les interlocuteur.ices de la diffusion du français en Palestine d'une part, et sur leur rapport à l'altérité d'autre part.

7 Les archives consulaires, tout au moins dans le cas de Jérusalem, sont constituées en grande majorité de correspondances émanant ou à destination des représentants du consulat. Il peut s'agir de correspondances politiques, et on trouvera dans ce cas des courriers échangés entre le ministère et les postes diplomatiques, mais également échangés sur un même territoire entre les consulats, les vice-consulats et les ambassades. Cela inclut en outre des échanges entre des postes français d'une même région, ou encore des correspondances commerciales. Je me suis intéressée en particulier aux échanges avec les écoles françaises ou les écoles locales ou étrangères proposant le français, mais aussi aux échanges avec les associations de diffusion du français (Alliance israélite universelle, Alliance française, Mission laïque française), avec les centres culturels français et les départements de français de différentes universités.

8 Les documents d'archives issus des postes diplomatiques constituent un matériau particulièrement riche pour appréhender - parfois par les silences (Perrot 1998) - la place des femmes dans les archives de la diplomatie linguistique. Contrairement aux archives diplomatiques conservées au centre de La Courneuve, qui correspondent aux archives centrales du ministère des Affaires étrangères, celles conservées à Nantes contiennent une richesse d'information qui n'a pas été lissée, standardisée, résumée. En effet, dans les échanges entre le ministère et les postes diplomatiques, seules les informations considérées comme particulièrement importantes remontent à l'échelon supérieur et souvent sous une forme épurée (formulaire, note de synthèse, etc.). Les archives de Nantes, c'est-à-dire celles des différents postes consulaires (ambassades, consulats, vice-consulats), incluent des dossiers de préparation à ces notes de synthèse qui comprennent le recensement d'articles de presse, de courriers d'acteur.ices du territoire (officiels, société civile, établissements privés). Ces différents documents donnent à voir de manière plus fine les points suscitant l'intérêt des autorités consulaires, l'étendue de leur connaissance sur le territoire et des partenariats, affinités, inimitiés entretenues avec les différent.es acteur.ices sur place. Cette image est étroitement liée à ce que le consulat dit, relève, note, consigne. Il est primordial de garder à l'esprit que c'est ce point de vue qui est archivé puis étudié. Cela peut parfois avoir pour effet d'invisibiliser ce que le consulat ne se représente pas. L'intérêt est de tâcher de comprendre comment les autorités consulaires se perçoivent, se mettent en discours, se représentent, au sein du réseau d'enseignement du français qu'elles décrivent. Les limites en sont que ce qui n'a pas été retenu par le consulat peut être perçu comme inexistant : la place des femmes en est un exemple significatif. On pourra ainsi participer à répondre aux questions posées par Michelle Perrot : "Quelles sont les représentations dominantes - ou marginales - que les hommes (car il s'agit d'eux presque exclusivement) ont données des femmes? Et comment les interpréter?» (Perrot 1998 : 377). 


\section{Archives de l'EPPFE}

En complément des archives diplomatiques, cette étude s'appuie sur les archives de l'École de préparation des professeurs de français à l'étranger. Inclure cette institution qui a préparé depuis 1920 des enseignant.es destiné.es à enseigner le français à l'étranger, en entretenant des liens étroits avec le MAE, permet de rendre compte de la diversité des formes qu'a pris la diplomatie linguistique. Les archives de l'EPPFE, qui font l'objet d'un travail d'indexation au sein du DILTEC, sont notamment composées de comptes-rendus de réunions, d'échanges avec les institutions partenaires (ministères, écoles et universités qui envoient ou reçoivent des étudiant.es-enseignant.es de l'école), mais aussi d'un riche bulletin de l'Amicale des ancien.nes étudiant.es. Ce dernier type de document présente un intérêt singulier pour interroger la place des femmes dans la diplomatie linguistique. D'une part, les bulletins donnent à voir les promotions d'étudiant.es-enseignant.es, et permettent de mesurer la part de femmes et d'hommes. D'autre part, ce bulletin est centré sur les nouvelles des ancien.nes et donne une idée des trajectoires des personnes passées par l'EPPFE. Enfin, ce bulletin rend compte d'une circulation des enseignant.es favorisant la constitution d'un réseau international encouragé par l'EPPFE. L'« Association amicale des anciens élèves de l'EPPFE », espace stratégique pour créer une communauté et un réseau d'influence, n'a pas fait l'objet d'étude à ce jour, bien qu'elle ait produit des documents qui donnent à voir des femmes et des hommes - en ce sens qu'y apparaissent étudiant.es et enseignant.es et non uniquement des dirigeants.

\section{Quelles implications dans la compréhension de la diplomatie linguistique?}

10 Cet article exploratoire propose des pistes de recherche pour interroger les archives afin d'évaluer la place des femmes dans la diplomatie linguistique. Où se situent les femmes dans ces deux strates d'archives de la diplomatie linguistique? Que nous disent ces archives des rôles joués par les femmes dans les stratégies d'influence? Que nous dit leur place sur le fait même d'archiver et sur les travaux d'histoire? Retracer la présence de femmes dans ces deux catégories d'archives peut en effet d'une part apporter un éclairage par le genre sur les rôles tenus dans la diffusion, et d'autre part sur la manière dont ces rôles sont considérés, archivés et mis en histoire. Chacune de ces pistes pourrait faire l'objet d'une étude à part et approfondie, l'ambition de cet article est d'esquisser des possibles et de pluraliser les représentations des femmes dans la diplomatie linguistique ${ }^{4}$.

\section{Les femmes, cibles au cœur des stratégies d'influence?}

11 Dans le cas des politiques d'influence en Palestine, les femmes ne sont pas absentes des archives diplomatiques. L'étude de ces archives officielles permet de comprendre le rôle qui leur est assigné au cœur même du processus d'influence sur les personnescibles. On conçoit ainsi que les écoles et la langue sont des passeuses de mentalité française, de culture française. Parmi les écoles, celles de jeunes filles représentent une cible toute particulière. Le vice-consul de Jaffa écrivant au consul de Jérusalem en 1912 exprime ainsi les raisons de l'importance des écoles de filles : 
Les mamans sont les premières éducatrices de l'enfance. Jeunes filles, leur intelligence s'est développée ici dans notre langue, et c'est pour ça qu'on peut voir de tout petits enfants de nationalité ottomane mais qui parlent français ${ }^{5}$. donnent à voir la part active prise par les femmes. Si elles sont au cœur des stratégies d'influence en tant que cibles, certaines sont aussi actrices de ces stratégies. C'est le cas des congrégations en Palestine. Les premières congrégations françaises à s'installer au Levant sont féminines ${ }^{6}$. Ainsi dès le milieu du XIX siècle, s'installent en terre inconnue, des Sœurs, des jeunes femmes formées et qui deviennent enseignantes. Maillons essentiels de l'enseignement en français au Levant, leurs échanges avec les institutions françaises font apparaitre une fine connaissance des jeux diplomatiques. Christophe Prochasson, chercheur en histoire des intellectuels, qualifie les correspondances de « chainon intermédiaire entre le texte et le contexte " (Prochasson 2009:2) qui peuvent nous «donner à voir les hiérarchies, les stratégies, les influences, les réseaux» (ibid.: 3). Les femmes membres des congrégations n'apparaissent que rarement et ces correspondances avec le consulat permettent en effet de percevoir leurs stratégies propres. extrait de l'introduction d'un courrier des Dames de Nazareth envoyé au consul général en 1936, pour demander qu'il intercède en leur faveur auprès du ministère :

Ici, nous faisons notre possible pour propager la langue française et faire aimer notre belle France. Nous avons eu une vraie déception cet été en constatant qu'une partie de nos subsides nous étaient enlevés?.

Les représentantes des congrégations savent que leur meilleur argument pour obtenir des financements réside dans l'enseignement de la langue et de la culture française et usent de cette corde sensible pour déplorer la baisse des subventions qui leur sont accordées.

Les archives diplomatiques consulaires permettent ainsi d'esquisser un tableau qui rend compte de l'action de femmes, bien que celles-ci n'aient pas eu accès aux fonctions plus visibles de diplomates. 
18 L'étude des bulletins de l'amicale de l'EPPFE permet également de rendre compte du grand nombre de jeunes femmes, souvent célibataires, exerçant comme enseignantes de français dans le monde entier (et ce dès les années 1930). Ce premier degré d'analyse permet de battre en brèche l'idée de femmes passives voire de femmes réceptacles des politiques d'influence. Les femmes qui apparaissent dans les archives de la diplomatie linguistique sont des actrices directes de l'enseignement du français à l'étranger - et par conséquent de l'influence - mais aussi des femmes stratèges dans l'emploi de la langue française comme levier de négociation avec les autorités.

19 Les archives de l'EPPFE permettent en outre de poser les jalons d'une étude sur un autre rôle peu valorisé alors même qu'il est au cœur des stratégies de l'école. Une recherche en cours ${ }^{8}$ s'intéresse à l'Amicale des anciens étudiants de l'EPPFE, créée au début des années 1920 pour consolider les liens entre l'école et ses étudiant.es sortant.es. Le bulletin que ce groupement publie à partir du milieu des années 1930 est d'une grande richesse pour identifier les trajectoires des étudiant.es de l'école, devenu.es enseignant.es dans le monde entier.

20 La figure de Mme Dufourcq permet de soulever la question de la valeur attribuée aux activités féminines. Secrétaire de l'École à partir de 1931, elle devient présidente d'honneur de l'Amicale. La fonction de ce groupement n'a pas fait l'objet d'étude avant les travaux en cours au sein du DILTEC. L'Amicale est pourtant pensée, dès sa création, dans une stratégie volontariste d'influence, et des femmes ont joué un rôle d'importance dans cette stratégie.

21 Dans l'histoire de l'École parisienne de formation d'enseignant.es de français à l'étranger, l'Amicale tient une place importante dans ce que les bulletins nomment «l'esprit de famille » ou " l'esprit d'école ». Le bulletin pourrait laisser penser qu'il ne s'agit que de prendre et donner des nouvelles aux étudiant.es passé.es par l'EPPFE. De fait, ces nouvelles occupent une part conséquente des bulletins. Quelques notes introductives ou commentaires permettent de saisir le rôle de cet espace éditorial: conserver les liens pour garantir des emplois par cooptation aux futur.es étudiant.es sortant.es, garantir de futur.es étudiant.es à l'école en diffusant son nom et ses méthodes à l'étranger, défendre l'école, sa qualité et ses diplômes auprès de partenaires et recruteurs...

22 Or, ce rôle est principalement tenu, animé, entretenu par des figures féminines, comme Mme Dufourcq, à la fois secrétaire de l'EPPFE et présidente d'honneur de l'Amicale dans les années 1930. Les témoignages et traces que l'on trouve dans les Bulletins ainsi que dans les rapports d'activités de l'EPPFE laissent apercevoir le rôle assidu joué par Mme Dufourcq, que ce soit dans l'organisation, la mise en relation, ou dans des interventions directes pour obtenir des postes à tel ou telle étudiant.e (comme cela est relaté dans le rapport annuel de 1938).

23 Il importe de porter un nouveau regard sur les rôles d'assistante, de secrétaire générale, de co-présidente qui sont peu traités dans les articles d'histoire et qui sont pourtant des socles incontournables des stratégies portées par les institutions. Dans le cas de l'EPPFE, "l'esprit d'école» a constitué un des moyens d'expansion et de visibilisation de l'institution. Quelle valorisation des personnes qui ont fait vivre cet esprit de communauté ? Quelle prise en compte du rôle central de femmes comme Mme Dufourcq dans la constitution des réseaux de diffusion, des réseaux d'influence, dans la circulation des personnes et des idées? 
24 L'EPPFE n'a pas fait l'objet d'un grand nombre de travaux, mais ceux-ci se sont concentrés sur des perspectives institutionnelles qui font principalement apparaitre les directeurs ou les recteurs en poste. Or, ces postes ne sont pas investis par les femmes ${ }^{9}$. Ainsi, en 1946, l'élection à l'Amicale voit uniquement des hommes candidats pour le poste de président, et uniquement des femmes candidates pour le poste de secrétaire, alors même que celles-ci sont très investies dans l'Amicale, plus nombreuses parmi les élèves, et qu'elles font vivre l'esprit de corps par les nouvelles envoyées chaque année.

Michèle Perrot nous alerte sur la « dévalorisation des activités féminines » (1998: IV), et cela semble être le cas pour le champ de la diplomatie linguistique où l'on ne prend pas la mesure du rôle joué.

De fait, les études existantes sur la diplomatie linguistique sont majoritairement centrées sur les institutions, et parmi elles sur les rôles de direction, presque tous tenus par des hommes jusqu'à très récemment. Il existe par conséquent un lien ténu entre les rôles professionnels et de pouvoir, les politiques d'archivages, et les travaux d'historien.nes. Cela implique que les recherches sur les archives traditionnellement mobilisées continueront à occulter la part jouée par de nombreuses femmes, tant que ne seront pas pris en considération d'autres prismes, d'autres matériaux et les potentiels points aveugles.

\section{Femmes en résistance}

Ces premières pistes permettent de prendre la mesure des missions menées par les femmes dans la diplomatie linguistique, peu documentées ou peu valorisées : missions d'enseignantes, de secrétaires. La sous-partie qui s'ouvre a pour objet de mettre en lumière des femmes qui ont transgressé leurs rôles en tant que femmes, sans que l'histoire ne les retienne pour cela. Les deux documents d'archives mobilisés ici, l'un issu des archives diplomatiques, l'autre des archives de l'EPPFE, sont importants dans la mesure où ils battent en brèche l'image de femmes dans leurs foyers et dociles aux idées des hommes. Michèle Perrot évoquait l'importance de ne pas effacer ces femmes qui sortent des rôles folkloriques, ruraux et tranquilles, que l'écriture de l'histoire a souvent préféré retenir.

\footnotetext{
Le risque est grand aussi d'enfermer une fois de plus les femmes dans l'immobilité des us et des coutumes, structurant le quotidien dans la fatalité des rôles et la fixité des espaces. Vision rassurante d'un monde rural sans conflits, le folklore est à certains égards la négation de l'histoire, une certaine manière de transformer en rites tranquilles les tensions et les luttes. Pourtant, ce sont les femmes en action qu'il importe de retrouver, innovant dans leurs pratiques, femmes animées, et non point comme des automates, mais créant elles-mêmes le mouvement de l'histoire. (Perrot 1998 : 154)
}

Le premier de ces documents est un extrait du bulletin de l'Amicale des anciens élèves de l'EPPFE de 1958 qui offre une double page au témoignage d'Irène Brunet. Partie pour la Jamaïque en tant qu'assistante de français et secrétaire de l'Alliance Française de la Jamaïque, elle s'interroge sur son rôle et la réception de son travail. Les premières lignes de son témoignage donnent à voir une part de sa personnalité, loin des stéréotypes.

Enfin le rêve de dix années devenait réalité. Partir. Connaitre d'autres mondes, une autre vie. Apporter au loin un peu de ce que j'imaginais être le vrai visage de la France. [...] J'avais peu de dons pour cette vie dite 
«coloniale » que l'on m'avait décrite : réceptions, danse, tennis, natation. Encore moins de don pour la vie en Société tout court, lorsque cette vie requiert sourire égal à chacun, sympathique ou non, diplomatie (hypocrisie ?) dans les actes et les paroles. Activités extra-universitaires? néant. "Hobby" ? rouler à toute vitesse sur une route droite et plate des Landes et m'arrêter à l'endroit le plus solitaire avec un bouquin.

\section{Brunet sur la diffusion du français qui interpellent :}

J'avais ingurgité pêle-mêle tout ce que deux années d'École, la préparation au CAPES, le stage m'avaient offert. [...] Très vite pourtant le travail s'est révélé hérissé de difficultés plus ou moins prévues. [...] Puis il a fallu adapter mes goûts personnels aux nécessités d'un programme, et réaliser soudain que je parlais à des étudiants d'Université qui avaient appris notre langue à l'école, mais qui étaient plus étrangers à notre culture que les petits élèves de $5^{\mathrm{e}}$ et $4^{\mathrm{e}}$ du Lycée Condorcet. Combien de fois ne me suis-je pas intérieurement arraché les cheveux devant un auditoire me regardant sans comprendre parce que je faisais allusion à des faits historiques, des manières de penser, des ouvrages contemporains ou des films que je supposais à tort universellement connus. [...] Mais ces difficultés qui obligent le nouveau venu à étudier l'histoire, la littérature du pays, ne sont que la découverte voulue, pleine d'intérêt d'un autre monde, l'approfondissement du travail d'enseignante que j'avais choisi. Bien plus nombreuses et désespérantes sont celles qui commencent avec la diffusion de la culture française, mission de l'Alliance. Comment diffuser la culture française avec des moyens plus que réduits, au sein d'une société qui s'en passe fort bien, qui n'a et n'a eu que des rapports très lointains avec la France, qu'une heureuse évolution politique vers l'indépendance ne peut qu'opposer à un colonisateur égoïste et belliqueux, continuellement en butte aux éléments qui voient dans l'Alliance un club mondain?

Si cet extrait s'inscrit dans une catégorie de discours ancrés dans l'époque coloniale, une prise de distance est opérée. Irène Brunet est consciente du décalage entre les ambitions d'une France diffusionniste et l'accueil réservé par une société jamaïcaine qui n'y voit guère d'intérêt. Cette remise en question du présupposé universaliste, qui voudrait que le monde attende de la France une intervention, fait écho aux propos d'une autre femme : Aminé Choufani.

31 Une trentaine d'années plus tôt, sur un autre continent, et depuis une position sociale (et mondiale) différente, cette jeune femme rejette les desseins coloniaux qui accompagnent l'ouverture de classes en Palestine. Dans les années 1920, alors qu'une école s'apprête à ouvrir à Bethléem, Aminé Choufani organise ainsi une conférence dont le contenu est retranscrit et conservé dans les archives diplomatiques du consulat de Jérusalem :

Il n'a pas suffi à l'occident de coloniser les terres, il envoie [s]es hommes pour coloniser les corps et les pensées en inculquant leurs idées au moyen des Écoles étrangères missionnaires.

Pour cela, il faut vous révolter devant toute autorité même religieuse et essayer de rebâtir notre gloire perdue en éduquant nos jeunes filles et nos jeunes gens.

J'ai honte et grand'honte de quelques-uns qui pour quelque vil intérêt ont empêché cette réunion. 
En face de cette action il vous faut dédaigner ces personnes qui veulent dominer sur nos pensées et nous rendre esclaves. ${ }^{10}$ Choufani » d'« illuminée » et raille le peu de succès de sa conférence.

La demoiselle Aminé Choufani, qui semble être une illuminée, avait déjà essayé de fonder une école [nationale] pour jeunes filles à Haïfa avec le but de soutirer les jeunes palestiniennes à l'influence des congrégations. N'ayant pas réussi à Haïfa, elle est venue à Bethléem et a donné une conférence pour essayer d'inculquer ses idées aux indigènes. Malgré une grande réclame pour attirer du monde à sa conférence, quelques personnes seulement y ont répondu, la population de Bethléem étant très divisée.

Après ce récent échec Mlle Choufani aurait l'intention d'essayer sa chance en Transjordanie. ${ }^{11}$ Pourquoi son contenu, sa tonalité m'avaient-elle tant marquée? Le texte de cette conférence venait compléter un type de traces conservées au sein des archives du consulat général de France à Jérusalem : celles des contestations. Le consulat semblait exercer une veille sur les réceptions de sa politique d'influence et conservait des articles de journaux, ou rapports de ses agent.es au sujet de différends, de menaces ou autres manifestations de désaccords. Elle m'avait intéressée dans le cadre de ma réflexion sur la relation à l'Autre, sur le regard posé sur les «indigènes ", sur la prétention à pouvoir transformer l'Autre par la diplomatie linguistique, sur la prétention à agir pour le bien de l'Autre voire à répondre à ses « attentes » :

Le moment est favorable et décisif, c'est un fait certain que la population de ces contrées, qu'elles soient juives, chrétiennes et j'oserais même dire musulmanes, manifestent à l'heure qu'il est une tendance naturelle à se soumettre à l'influence de la France et à en accepter la langue et les idées. Nous avons donc le droit et le devoir, si nous voulons rester fidèles à la 
tradition de notre politique orientale d'aider ces tendances à se dégager et de les diriger dans une voie utile $\mathrm{e}^{12}$. (Perrot 1998: introduction), les pistes explorées dans cet article permettent de réfléchir à ce qui a été archivé sans pour autant faire histoire. L'appel à contributions de ce numéro appelait à nous interroger sur la manière dont le discours historique produit le sens de l'archive. Quelles sont les traces (Veyne 1996) que l'on garde des femmes et quelles sont celles que l'on transforme en histoire?

42 récente, les femmes ont pourtant percé dans certains types d'archives (consulaires, bulletins associatifs), reste alors aux historien.nes de se saisir de ces rôles jusque-là non considérés, pour reécrire l'histoire d'institutions comme le consulat ou l'EPPFE. Proposer des relectures, en s'appuyant sur le sens toujours ouvert de ces traces (Dosse $2001: 140)$. 


\section{BIBLIOGRAPHIE}

CABANEL, Patrick. 2006. Une France en Méditerranée : écoles, langue et culture françaises, XIX $\mathrm{XX}^{\mathrm{e}}$ siècles. Grâne : Créaphis.

GAZEAU-SECRET, Anne. 2013. «"Soft power" : l'influence par la langue et la culture » Revue internationale et stratégique 89 (1) : 103-110.

DOSSE, François. 2001. « Le moment Ricœur » Vingtième siècle, revue d'histoire 69 (1) : 137-152.

DOSSE, François. 2003. « Michel de Certeau et l'écriture de l'histoire » Vingtième Siècle. Revue d'histoire 78 (2) : 145-156.

DOSSE, François. 2006. Paul Ricœur et Michel de Certeau : l'histoire, entre le dire et le faire, Paris : L'Herne.

KoK-ESCALLE, Marie-Christine. 2012. " Les discours de et sur la femme éducatrice dans les ouvrages pour l'enseignement du français aux Pays-Bas (XVI ${ }^{\mathrm{e}}-\mathrm{XIX}^{\mathrm{e}}$ siècles) » Documents pour l'histoire du français langue étrangère ou seconde 47-48 : 137-154.

OFFENSTADT, Nicolas. 2010. « Archives, documents, sources ", in Historiographies I, concepts et débats, DelACroIX Christian et al. (éd.). Paris : Gallimard, 68-78.

PERROT, Michelle. 1998. Les femmes ou Les silences de l'histoire. Paris : Flammarion.

PROCHASSON, Christophe. 1991. «Les correspondances : sources et lieux de mémoire de l'histoire intellectuelle » Les Cahiers du Centre de Recherches Historiques. [En ligne], 8 | 1991, mis en ligne le 18 mars 2009, consulté le 10 décembre 2020. URL : http://journals.openedition.org/ccrh/2824 ; DOI : https://doi.org/10.4000/ccrh.2824

RECLUS, Onésime. 1899. Le plus beau royaume sous le ciel. Paris : Hachette.

RUBIO, Clémentine. 2018. Une langue en mission, histoire des politiques linguistiques et didactiques françaises en Palestine, Thèse de doctorat, Université de Tours.

RUBIO, Clémentine. 2021 (à paraitre), L'enseignement du français en Palestine, d'après les archives diplomatiques du consulat général de France à Jérusalem. Limoges : Lambert-Lucas.

SPAËTH, Valérie. 1998. Généalogie de la didactique du français langue étrangère : l'enjeu africain. Paris : CIRELFA-Agence de la francophonie : diff. Didier érudition.

SPAËTH, Valérie. 2010. « Mondialisation du français dans la seconde partie du XIX ${ }^{\mathrm{e}}$ siècle :

l'Alliance Israélite Universelle et l'Alliance Française » Langue française 167 (3).

VERDEIL, Chantal. 2014. «La sœur, le prêtre et les mariés : lecture genrée d'une source missionnaire (1936) » in Genre et christianisme, Plaidoyers pour une histoire croisée, BRÉJON DE LAVERGNÉE Matthieu, DELLA SUDDA Magali (éd.). Paris : Bibliothèque Beauchêne, 127-151.

VEYNE, Paul. 1996. Comment on écrit l'histoire. Paris : Seuil.

\section{NOTES}

1. Voir par exemple Cabanel 2006 ; Rubio 2018 ; Spaëth 1998, 2010.

2. Société internationale pour l'histoire du français langues étrangère et seconde. 
3. Projet de l'équipe de recherche DILTEC visant à créer des ponts entre les chercheur.es de didactique des langues qui s'intéressent à l'histoire.

4. Plusieurs chercheuses ont d'ores et déjà produit des travaux de recherche sur le sujet des femmes dans le champ de la diffusion des langues, souvent à partir du cas des congrégations (et moins par le prisme diplomatique). On peut nommer Chantal Verdeil ou Marie-Christine KokEscalle. Le numéro de la revue Documents pour l'Histoire du Français Langue Etrangère et Seconde de $2012\left(n^{\circ} 47-48\right)$ porte sur les femmes, principalement à travers les manuels qui s'adressent à elles ou à leurs représentations.

5. CADN, Jérusalem, Série A, dossier 118, Subventions. Courrier du vice-consul de Jaffa au consul (1912).

6. Entre 1848 et 1870 , les trois congrégations pionnières sont féminines : les Sœurs de Saint Joseph de l'Apparition, les Sœurs de Nazareth et les Sœurs de Notre-Dame de Sion.

7. CADN, Jérusalem, Série B, dossier 188 (1936).

8. Géopolitique des trajectoires enseignantes : circulation des personnes et des concepts, Alice Burrows et Clémentine Rubio. Projet de recherche mené à partir des archives de l'EPPFE et visant à étudier les trajectoires professionnelles des membres de l'école afin de comprendre comment ces circulations dessinent une histoire des contacts entre les espaces académiques, tout en dressant une géopolitique des rapports académiques et (donc) des rapports politiques.

9. Par règlement ou par coutume?

10. CADN, Jérusalem, Série B, dossier 186 (document non daté, autour des années 1920).

11. CADN, Jérusalem, Série B, dossier 186 (document non daté, autour des années 1920).

12. CADN, Jérusalem, Série A, dossier 138. Courrier du consul au ministre (1879).

13. Il importe de prendre en compte également que les trajectoires des femmes s'effacent quand elles se marient : l'étude en cours sur les archives de l'EPPFE mène par exemple à de nombreuses impasses. Lorsque l'on essaye de retrouver les trajectoires étudiantes puis enseignantes des femmes et qu'une Mary Ling devient Mrs Borgard (bulletin de l'Amicale de l'EPPFE, 1946), son histoire est découpée et disparait en quelque sorte.

\section{RÉSUMÉS}

Cet article s'attachera à esquisser des pistes de recherche sur les femmes dans les archives de la diplomatie linguistique au tournant $\mathrm{du} \mathrm{XIX}^{\mathrm{e}}-\mathrm{XX}^{\mathrm{e}}$ siècle. À partir de deux types d'archives, les archives diplomatiques émanant du consulat général de France à Jérusalem et les archives de l'École de préparation des professeurs de français à l'étranger, il s'agira de comprendre la place attribuée aux femmes et les rôles joués par les femmes. Cette analyse s'appuiera à la fois sur les traces effectives des femmes dans les archives et sur des questionnements épistémologiques sur la mise en récit des traces et des absences.

This article identifies research perspectives with regards to women in archives of linguistic diplomacy. Based on the study of two types of archives, diplomatic archives form the French general consulate in Jerusalem and the archives of the "École de préparation des professeurs de français à l'étranger", this article examines the roles assigned to and played by women. This analysis draws upon effective traces of women in the archives and upon epistemological considerations concerning the creation of a narrative based on these traces and absences. 
INDEX

Thèmes : Explorations

Keywords : diplomatic archives, linguistic diplomacy, women, EPPFE, promotion of the French language

Mots-clés : archives diplomatiques, diplomatie linguistique, femmes, EPPFE, diffusion du français

\section{AUTEUR}

\section{CLÉMENTINE RUBIO}

Docteure en sociolinguistique et didactique des langues, Clémentine Rubio étudie l'histoire politique de la diffusion du français et les conceptions françaises de l'altérité. Ses recherches récentes portent sur les parcours de femmes et d'hommes, étudiant.es et enseignant.es, dont les trajectoires dessinent des rapports de force politiques et sociaux. Parmi ces rapports de force, la question du genre est étudiée dans ses implications épistémologiques et socio-professionnelles. 